\title{
Study on Cause Analysis and Key Technologies of Defect Prevention for Xiamen BRT Facilities under Complex Environment
}

\author{
Yichun Huang ${ }^{1}$, Wangtu $\mathrm{Xu}^{2^{*}}$ and Qifeng Cheng ${ }^{1}$ \\ ${ }^{1}$ Holsin Engineering Consulting Co., Ltd, Xiamen 361009, Fujian, China \\ ${ }^{2}$ School of Architecture and Civil Engineering, Xiamen University, Xiamen 361005, China; $\mathrm{PH}(86)$ \\ 592-2188958; FAX (86) 592-2188958 \\ ato1981@xmu.edu.cn \\ *The corresponding author
}

Keywords: Transport infrastructure; Bus Rapid Transit (BRT); Facility defect; Cause analysis; Defect prevention; Xiamen City

\begin{abstract}
In this paper, we examine the cause of facility defect of Bus Rapid Transit (BRT) services of Xiamen City, China. The cause analysis is carried out by using the Factor Analysis Approach (FAA) for determining the reason of the facility defects under complex environment. The complex environment is defined with six-dimensional factors which include the weather induced, bus operation caused, artificially induced improper structure induced and aged material induced. Based on the cause analysis, key technologies for detecting and preventing BRT defects are proposed. It hopes that this paper could provide a valuable reference to the maintenance of BRT facilities for cities which own the BRT system.
\end{abstract}

\section{Introduction}

Bus Rapid Transit (BRT) is a typical public transport means, which was introduced to many cities all over the world. It is a representative system which is responsible for carrying the medium-scale passenger volume and making up the transportation mission for the large-scale metro transit system (Chen, Yu, Yu, \& Guo, 2007). During the past 20 years, many foreign companies had propagandized to confuse a large number of Chinese cities to build BRT systems, e.g., Beijing, Hangzhou, Guangzhou, Xiamen, Changzhou, and Kunming (W. Wang, Yun, \& Yang, 2015). After constructing of these BRTs, let alone the function on optimizing urban transport systems, the experiences for maintain and management the engineering facilities are very limited - which result in the situation that many cities reluctantly agree to pay the high cost to maintain or repair these systems (Y. Wang, et al., 2013). Even worse, there always lacks the experiences for BRT maintenance globally (Suisman, 2010). This fact directly increases the difficulty of BRT system management. Consequently, many Chinese cities, such as Xiamen and Kunming, had to discard some built BRT routes or stations, to keep the balance between the fare income and the maintenance expense (XiamenBRTCooperation, 2015).

However, for the operating BRT systems, they still need the related technologies to reduce the maintenance cost and keep the system operating in good order, then to ensure the safe operation of the whole public transport system. Under this circumstance, this paper seeks to determine the main reason which results from the facility defects or called the diseases of the structure of BRT system. Based on the cause analysis, we proposed the key technology to detect these defects and provided the measure for avoiding them.

The rest of this paper has been organized as follows. Section 2 demonstrates the background of Xiamen BRT and the resultant defects. Section 3 proposes the methodology to determine the cause of BRT defects. Section 4 presents the method for factor effect determination. Section 5 details the key technology to detect and avoid these defects. Section 5 concludes. 


\section{Background}

Xiamen BRT is the only one overhead BRT system in China. It was opened to operation on September 1st, 2008. The biggest feature is the Xiamen BRT's construction is that it is fully viaduct on the Xiamen Island (CBD of Xiamen City), and is set up with the closed-form special lanes, to ensure rapid transit has the full closure of the proprietary right, which overcomes the drawbacks of interfering with other vehicles and pedestrians (Xiamen BRT Cooperation, 2015).

As the bridge age increased, influenced by climate, oxidation, corrosion and other environmental factors, and long-term steady and alive loads on the system (earthquake, typhoon, car, etc.), different degrees of damage and sudden damage on the BRT bridge structures or components occurred in recent years. Under the impacts of the defects, the strength and stiffness of BRT bridge structures or components will necessarily decrease with time. Moreover, the performance of the structure will be gradually deteriorated, thereby endangering the safety of the bridge (XiamenBRTCooperation, 2015).

Given the particularity of maintenance of BRT systems, there lacks the mature experience all over the word. Due to that, the global BRT systems are almost younger than 15 years old; there is not the overall standard or manual for BRT maintenance to date. For Xiamen BRT, due to its particular right of way (ROW) and system constructions, it is even much difficult to maintain the whole system. According to the record of Xiamen BRT Operation Cooperation, the annual maintenance cost for the entire BRT system of Xiamen City all exceeded 0.5 billion RMB Yuan in the recent three years (Xiamen Holsin Engineering Testing Co., 2014a).

Hence, it is urgent to determine the primary cause of the deficiencies on BRT structures and constructions. From saving maintenance cost, it is also necessary to find out the most flexible technologies for disease prevention for the BRT system. To solve this problem, this paper examines the factors which result in defects on BRT bridges. The related factors have been classified into six dimensions, which consists of the "complex environment." Based on the cause of BRT defects, the key technologies for defect prevention is presented accordingly. It hopes that the experience presented in this paper could provide a supportive reference for the BRT facility maintenance and safe operation of the city which is operating BRT all over the world.

\section{Definition of the "Complex Environment"}

What exactly is a "complex" environment in the construction \& building filed has recently challenged the knowledge of many scholars and engineers? Always exposure to air, coupled with high-intensity use, human-made damage and another random effect, the BRT would necessarily lose its original function gradually. To systematically determine the factors which affect the use age of BRT components, the related deficiency data observed from Xiamen BRT system between 2013 and 2014 have been shown in Table 1.

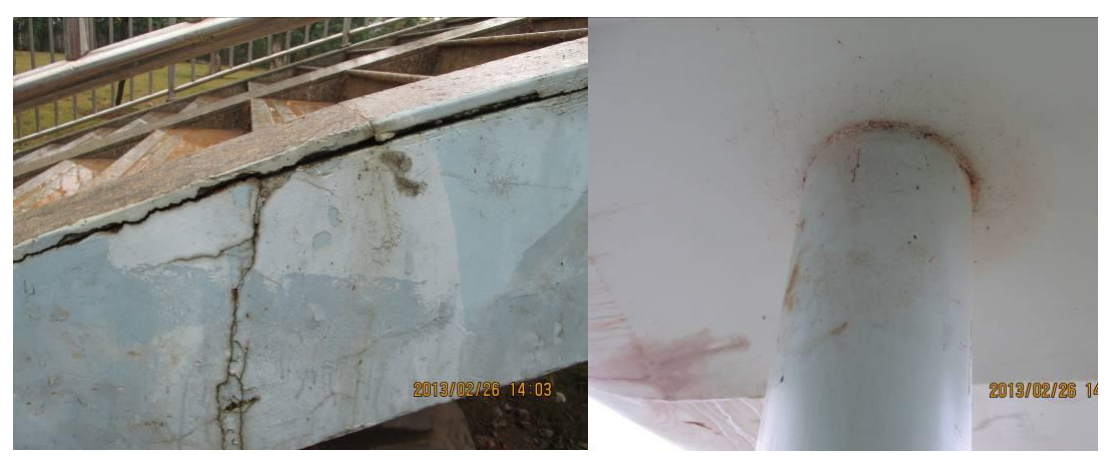

a Cracks on the steps

b. Corrosion on the support column 


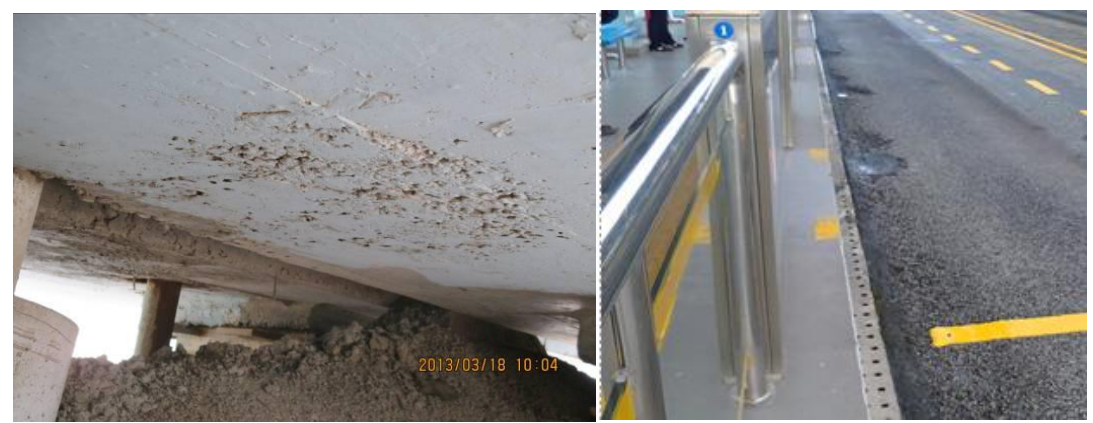

\section{c. Bridge pier settlement}

d. Rutting and potholes on the surface

Figure 1. Examples of deficiencies on Xiamen BRT structures and components (Xiamen Holsin Engineering Testing Co., 2014b)

According to those defect data, we summarize the defects on BRT structures and components, which have been shown in Table 1.

Table 1 Sample data of defects on Xiamen BRT structures and components

\begin{tabular}{|c|c|c|c|c|}
\hline Defect \# & & Structure or Component & Length of defect $(\mathrm{cm})$ & Possible cause detection \\
\hline & 1 & Bridge surface & 20 & Man-made sabotage \\
\hline \multirow{3}{*}{$\ldots$} & 2 & Railing & 30 & Weather \\
\hline & & $\ldots$ & $\cdots$ & $\ldots$ \\
\hline & 5000 & Support & $\ldots$ & Vehicle operation \\
\hline
\end{tabular}

Using the defect sample data as shown in Table 1, the main causes of the causes which result in the BRT defects could be classified into six types as illustrated in Fig. 2.

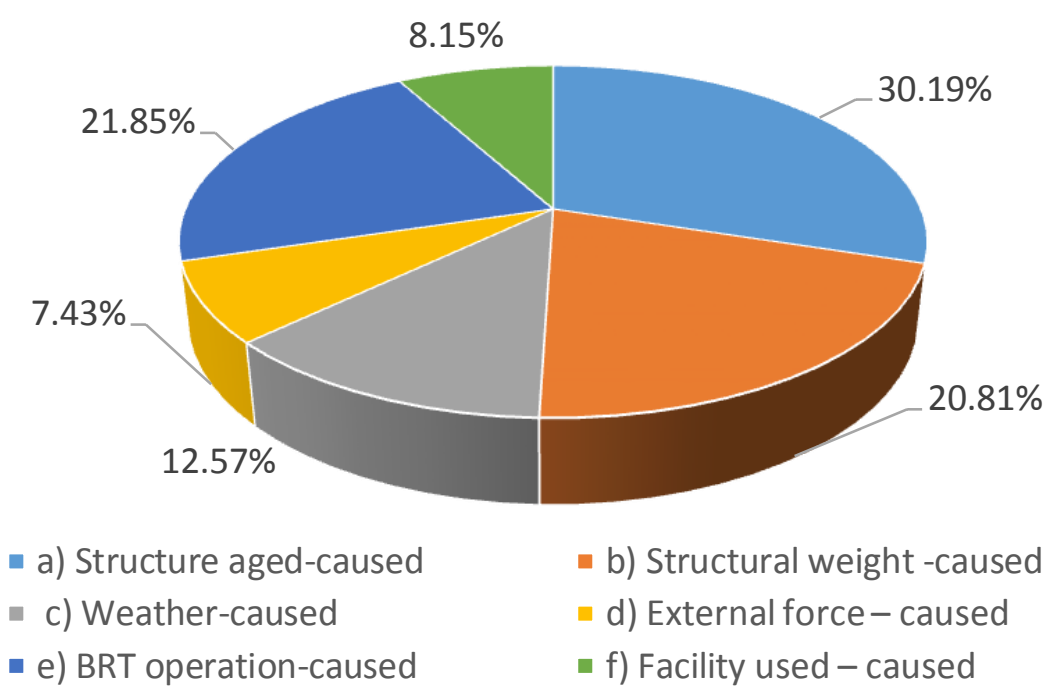

Figure 2. Primary cause of defects on Xiamen BRT structures and components (Xiamen Holsin Engineering Testing Co., 2014a)

Based on the statistical result in Fig. 2, we define the "complex environment" of BRT system as 
follows: The "complex environment" of BRT system denotes the external conditions or factors which result in the dysfunction of BRT structure or component. The related environmental inputs include the structure aged factor, structure weight factor, weather factor, external force factor, BRT operational factor and facility used factor.

\section{Determine the Cause Effects of BRT Construction Deficiencies}

The Used Method. Although that we have given out the statistical result of the six leading causes of defects on BRT structures and components, the detail effect of the each cause is still not clear. For probing into the effect of each cause, the Factor Analysis Approach (FAM) is adopted.

Based on the analysis of the relationship between the scan index and its influencing factors, FCA is used to determine the influence degree of each related factor quantitatively. FCA can not only comprehensively analyze the impact of various factors on an individual situation, but also examine the influence of some factors on the case individually (Park, Lord, \& Wu, 2016).

Mathematically, the main steps of FCA are as follows (Ramos-Quintana, et al., 2016; Zhou, et al., 2016).

Assuming that the subject index $\mathrm{M}$ is caused by three inter-related factors $\mathrm{A}, \mathrm{B}$, and $\mathrm{C}$. The multiple effects of these three factors could be represented as the multiplication relationships. Moreover, the analytical periods are divided into the reporting period (RP) and the planning period (PP).

Accordingly, the RP index could be denoted: as $M_{1}=A_{1} \times B_{1} \times C_{1}$; further the PP index could be represented as: $M_{0}=A_{0} \times B_{0} \times C_{0}$.

When determining the influence degree of each factor on the change of index $M$, the Serial Iterative Method (SIM) could be used. The procedures are:

Calculated the PP index:

$M_{0}=A_{0} \times B_{0} \times C_{0}$

The first iteration: Replace the first factor and calculate the subject index $\mathrm{M}$ again:

$M_{1}=A_{1} \times B_{0} \times C_{0}$

The second iteration: Replace the second factor and calculate the subject index M again:

$M_{2}=A_{1} \times B_{1} \times C_{0}$

The third iteration: Replace the third factor and calculate the subject index M again:

$M_{3}=A_{1} \times B_{1} \times C_{1}$

Calculate the effect of each factor as follows:

$$
\begin{aligned}
& E_{A}=M_{1}-M_{0} \\
& E_{B}=M_{2}-M_{1} \\
& E_{C}=M_{3}-M_{2}
\end{aligned}
$$

Calculated the normalized effect:

$$
E_{A}^{\prime}=\frac{E_{A}}{\max \left(E_{A}, E_{B}, E_{C}\right)}
$$




$$
\begin{aligned}
& E_{B}^{\prime}=\frac{E_{B}}{\max \left(E_{A}, E_{B}, E_{C}\right)} \\
& E_{C}^{\prime}=\frac{E_{C}}{\max \left(E_{A}, E_{B}, E_{C}\right)}
\end{aligned}
$$

Compute the overall effect of all factors:

$$
\Delta E=E_{A}^{\prime}+E_{B}^{\prime}+E_{C}^{\prime}
$$

If the number of factors is larger than three, the iterative procedure is in the same manners.

Method Applications. In this section, we used the number of defects which caused by the six factors to examine the factor effect. The subtotal of BRT defect numbers by the six factors

\begin{tabular}{|c|c|c|c|c|c|c|c|c|c|c|c|c|}
\hline \multirow{2}{*}{$\begin{array}{l}\text { Index } \\
\text { Year }\end{array}$} & \multicolumn{2}{|c|}{$\begin{array}{l}\text { a) Structure } \\
\text { aged }\end{array}$} & \multicolumn{2}{|c|}{$\begin{array}{l}\text { b) Structural } \\
\text { weight }\end{array}$} & \multicolumn{2}{|c|}{ c) Weather } & \multicolumn{2}{|c|}{$\begin{array}{l}\text { d) External } \\
\text { force }\end{array}$} & \multicolumn{2}{|c|}{$\begin{array}{c}\text { e) BRT } \\
\text { operation }\end{array}$} & \multicolumn{2}{|c|}{$\begin{array}{l}\text { f) Facility } \\
\text { used }\end{array}$} \\
\hline & 2013 & 2014 & 2013 & 2014 & 2013 & 2014 & 2013 & 2014 & 2013 & 2014 & 2013 & 2014 \\
\hline Factor & $A_{0}$ & $A_{1}$ & $B_{0}$ & $B_{1}$ & $C_{0}$ & $C_{1}$ & $D_{0}$ & $D_{0}$ & $E_{0}$ & $E_{1}$ & $F_{0}$ & $F_{1}$ \\
\hline $\begin{array}{l}\text { Caused } \\
\text { defect } \\
\text { number }\end{array}$ & 3031 & 4272 & 808 & 1129 & 1500 & 1300 & 625 & 625 & 2320 & 2705 & 1022 & 3210 \\
\hline
\end{tabular}
presented above have been shown in Table 2 .

Table 2 The subtotal of BRT defect numbers by the six factors presented in Section 3

Based on the data shown in Table 2, we could calculate the individual effect of the factor according to FAA shown above.

Table 3 The individual effect of each factor as well as the comprehensive effect

\begin{tabular}{lcc}
\hline \multicolumn{1}{c}{ Factor (s) } & Increase defect number & Individual effect \\
\hline a) Structure aged & 1241 & 0.096 \\
b) Structural weight & 321 & 0.131 \\
c) Weather & -200 & -0.062 \\
d) External force & 0 & 0.000 \\
e) BRT operation & 385 & 0.066 \\
f) Facility using & 2188 & 1.000 \\
Overall effect & 3935 & 1.232 \\
\hline
\end{tabular}

Based on Table 3, it is evidenced that the factor which owns the largest effect on the BRT defects is the facility using. On the other aspect, the weather has the least impact on BRT structure defects. Further, the external force has the negotiable effect to the occurrence of BRT construction defects. This result is out of the extent of the experiencing judgments - which attribute the main reasons of the defect of Xiamen BRT to the structure age and weather (Xiamen Holsin Engineering Testing Co., 2014b). Hence, we think the present result could help to adjust the judgment.

\section{Key Technologies to Prevent the BRT Structure Defects}

Asphalt pavement, rutting, wear, loose, potholes and other serious diseases, affecting the safe 
operation of traffic while increasing the BRT bus wear and tear (MOT of China, 2011). These defects should need a periodical, large area of repair, in a limited window timetable. As a result, it needs developing the new BRT surface concrete material which could be used in good condition.

As the bridge expansion joints often damage, it needs routine repair. However, a bridge expansion joints repair usually takes a month of replacement time, and occupies half of the lane, which led to the operation of the bus for up to a month's time inside, seriously affect the safe operation of BRT. For this consideration, to prevent the secondary damage to the BRT structure, we suggest replacing the bridge expansion joint with a half a year period.

Due to the construction of the rush period, resulting in the uneven settlement has not yet completed the frame beam, some bridges would produce a substantial shear force, which makes the bridge bearing different degrees of damage, or even offset. For this consideration, we have elopement the digital fiber under the supports of columns of BRT bridge, to detect the settlement time to time. On the other hand, we should propose the experienced formulation to calculate the settlement of the supports of BRT bridges. Using the computational result of settlement via the experienced formulation, the data could be compared with those obtained by the fiber detector, to examine the relationship between the differences, which could keep the reasonable settlement record of the BRT bridge.

For the conservation and management of pavement, at present only the way of visual maintenance decision-making, there is no system of road surface quality monitoring data for the maintenance of pavement management support. Hence, we should add a system to visualize the maintenance data of the BRT surface.

For typhoons, earthquakes, and other inclement weather natural disasters, there are no effective monitoring and surveillance tools for early warning, timely detection of extreme weather conditions could affect the safe operation of means.

For the maintenance of the road, the only way to carry out maintenance of visual decision-making, there is no system of road surface quality monitoring data for the BRT road maintenance management.

\section{Conclusion}

In this paper, we propose the definition of the environmental inputs which result into the deficiencies on BRT structures and components. Using Xiamen BRT as an example, a six-dimensional factor is formulated to determine the leading causes of BRT defects. Using the factor analysis approach, the effect of the individual cause of BRT defects is commutated. Based on the factor effect, the key technologies to prevent the defects on BRT structures and components are proposed.

Since Xiamen BRT has its unique right of way and the overhead configuration, the techniques presented in this paper still have their imperfections. However, we still hope they could be used as references for the BRT maintenance of other cities.

\section{Acknowledgement}

This work is supported by Xiamen Science and Technology Project under Grant Number of $3502 Z 20153001$.

\section{References}

[1] Chen, X. M., Yu, L., Yu, L., \& Guo, S. X. (2007). Impacts of Exclusive Lanes and Signal Priority on Bus Rapid Transit Effectiveness. International Conference on Transportation Engineering 2007 pp. 364-369).

[2] MOT of China (2011). "Highway bridge technical condition assessment standard" (H21-2011 JTG/T). People Communication Press. 
[3] Park, B.-J., Lord, D., \& Wu, L. (2016). Finite mixture modeling approach for developing crash modification factors in highway safety analysis. Accident Analysis \& Prevention, 97, 274-287.

[4] Ramos-Quintana, F., Guerrero, J. A., Rizo-Aguilar, A., Urzua-Vázquez, E., Hernández-Rabadán, D. L., \& Mayorga, P. P. (2016). A novel approach for assessing factors affecting biodiversity based on networks analysis. Ecological Indicators, 70, 460-465.

[5] Suisman, D. (2010). The Los Angeles Metro Rapid System: Designing Rapid Bus Service From the Sidewalk Up. Urban Public Transportation System pp. 153-158).

[6] Wang, W., Yun, M., \& Yang, D. (2015). Research on Method of Urban Traffic Mode Split Forecast in the Case of Introduction of Bus Rapid Transit. CICTP 2015 pp. 1358-1366).

[7] Wang, Y., Wang, Z., Li, Z., Staley, S. R., Moore, A. T., \& Gao, Y. (2013). Study of Modal Shifts to Bus Rapid Transit in Chinese Cities. Journal of Transportation Engineering, 139, 515-523.

[8] Xiamen BRT Cooperation (2015). Xiamen BRT Detection Report. pp. 1-150). Xiamen: Xiamen BRT Cooperation.

[9] Xiamen Holsin Engineering Testing Co., L. (2014a). BRT bridge bearing capacity simulationXiamen City Bus Rapid Transit System (BRT) analysis report- B section. Xiamen: Xiamen BRT Cooperation.

[10] Xiamen Holsin Engineering Testing Co., L. (2014b). Platform health inspection report- Xiamen City Bus Rapid Transit System (BRT) analysis report- B section. Xiamen: Xiamen BRT Cooperation.

[11]Zhou, D., Ning, Y., Wang, B., Wang, G., Su, Y., Li, L., \& Wang, Y. (2016). Study on the influential factors of $\mathrm{Cd} 2+$ on the earthworm Eisenia fetida in oxidative stress based on factor analysis approach. Chemosphere, 157, 181-189. 